\title{
Aerosol Box Decreases Aerosol Exposure only in Depressurized Rooms on Intubation and Extubation
}

Wataru Sakai ( $\nabla$ sakaiwataru1128@gmail.com )

Sapporo Medical University

Gen Hasegawa

Sapporo Medical University

Tomohiro Chaki

Sapporo Medical University

Shunsuke Tachibana

Sapporo Medical University

Michiaki Yamakage

Sapporo Medical University

\section{Research Article}

Keywords: Aerosol Dispersal, Aerosol Generated Procedure, Aerosol Box, Aerosol Exposure

Posted Date: December 30th, 2020

DOI: https://doi.org/10.21203/rs.3.rs-134254/v1

License: (c) (i) This work is licensed under a Creative Commons Attribution 4.0 International License.

Read Full License 


\section{Abstract}

The aim was to assess the effectiveness of an aerosol box objectively in several clinical settings with our experimental model.

Cake flour as an aerosol model was expelled with our experimental cough model. The primary outcomes were the number of 0.3-10- $\mu \mathrm{m}$-sized particles. Secondary outcomes were high-resolution videography of aerosol dispersal during intubation and extubation with an aerosol box in a pressurized or depressurized room.

On post hoc analysis, counts of all particle sizes at the physician position, medical staff position, and in the environment were significantly lower in a depressurized room with an aerosol box than in a pressurized room without an aerosol box during intubation ( $p<0.05$ in all situations and all particle sizes). Counts of all particle sizes at the physician and medical staff positions were significantly lower in the depressurized room with an aerosol box than in a pressurized room without an aerosol box during extubation ( $p<0.05$ in both situations and all particle sizes). Visual assessments supported the results of the primary outcomes and showed that an aerosol box in a depressurized room could effectively decrease aerosol exposure of the physician, medical staff, and environment.

In conclusion, an aerosol box decreased aerosol exposure only in a depressurized room.

\section{Introduction}

During this SARS-CoV-19 pandemic, physicians must intubate or extubate patients even if they cannot completely assess whether the patient has any infectious diseases. Because polymerase chain reaction testing and thoracic computed tomography cannot completely diagnose all cases of SARS-CoV-19 infection,[1] we should prevent aerosol dispersal as much as possible with every patient during aerosolgenerating procedures. Moreover, even when this pandemic subsides, a new and/or unknown infectious disease that can be transmitted by aerosolized particles is likely to appear. Thus, an effective way to assess and prevent aerosol dispersal during aerosol-generating procedures is needed.

There has been no clear evidence about aerosol dispersal during aerosol-generating procedures, because aerosol spread is affected by the kind of aerosol-generating procedure and environmental conditions, including room air flow, temperature, and humidity, which all differ from hospital to hospital.[2] Since infectious aerosols are invisible to the naked eye, it is nearly impossible to confidently assert that the aerosol protection being used is truly effective. It is difficult to assess aerosolized particles individually because quantitative, temporal, and spatial (three-dimensional) assessment of aerosol dispersal is essential, but there has been no clear way to do so.

An aerosol box is currently the most well-known and effective aerosol barrier during intubation. Several studies have shown its effectiveness.[3,4] However, a recent report questioned whether an aerosol box 
could decrease the risk of infection.[5] Because of the difficulty of assessing aerosol dispersal, the effectiveness of an aerosol box has not been determined conclusively.

The present study was conducted to clarify whether an aerosol box could prevent aerosol spread under several conditions during intubation and extubation, with particle counting and our novel threedimensional particle visualizing method, which could provide quantitative, temporal, and spatial information regarding aerosol dispersal. In addition, a novel three-dimensional particle visual assessment method with common items is introduced, which can provide physicians worldwide the ability to assess aerosol dispersal independently.

\section{Materials And Methods Cough Model}

An Advanced Life Support training mannequin (Laerdal Medical, Tokyo, Japan) was used as a lung and airway model. The stomach of the mannequin was detached, and the esophagus of the mannequin was completely closed with vinyl tape to prevent air leakage. Both lungs of the mannequin were massaged simultaneously, as shown in Supplemental Video 1, and the air in the lungs was expelled out of the mouth of the mannequin, which was defined as the artificial cough model. The parameters of the artificial and human coughs in the supine position were measured with a particle image velocimetry system (PIV) (Kato Koken, Kanagawa, Japan) and spirometry (MICROSPIRO HI-302, NIHON KOHDEN, Tokyo, Japan) in the preliminary study. (Table 1 and Supplemental Video 1).

Table 1

Environmental factors of the simulation and the artificial cough patterns

\begin{tabular}{|c|c|c|}
\hline Environmental factor & & \\
\hline Air change rate $(/ \mathrm{h})$ & & 35 \\
\hline Fresh air change rate $(/ \mathrm{h})$ & & 3 \\
\hline Room size (length'width'height, m) & & $5.1^{\prime} 5.1^{\prime} 3$ \\
\hline Temperature $\left({ }^{\circ} \mathrm{C}\right)$ & & $22-27$ \\
\hline Humidity (\%) & & $50-55$ \\
\hline Perpendicular wind speed (m/s) & & 0.35 \\
\hline Atmospheric pressure in depressurized/ pressurized pressure room $(\mathrm{Pa})$ & & $\leq 2.5 />2.5$ \\
\hline Parameters of human and artificial coughs in the preliminary study & Human & Artificial \\
\hline Maximum speed around the mouth & 10 & 10 \\
\hline Duration (seconds) & 0.05 & 0.1 \\
\hline Volume (ml) & $<940$ & $410-450$ \\
\hline
\end{tabular}




\section{Tracers}

The diameter of cake flour particles was $2.5-280 \mathrm{~mm}\left(\mathrm{D}_{50}=25.56 \mu \mathrm{m}\right)$, which was measured with a particle counting device (LA-950, HORIBA, Ltd., Kyoto, Japan). Before each experiment, $2 \mathrm{~g}$ of cake flour were placed into the mannequin's mouth (Supplemental Video 1).

\section{Particle counting assessment}

Aerosolized particles with diameters of $0.3,0.5,1.0,2.5,5.0$, and $10 \mu \mathrm{m}$ were measured with a particle counting device (Model P8-306, Airy Technology Japan Ltd., Tokyo, Japan). Particle counting was started after confirming that the aerosolized particle level was under the set baseline $(2.5-\mu \mathrm{m}$-diameter particle count $<1400$ ) and done for one minute before and after the artificial cough to calculate aerosol exposure as the delta between two values. The device was placed in three locations, around the physician's face for the doctor's aerosol exposure, the medical staff's face (right side of the mannequin) for the nurse's aerosol exposure, and at the foot of the operating bed for the environmental aerosol exposure.

\section{Particle Visualization and Image Capture}

A projector (EB-1985WU, Epson, Nagano, Japan) as a strong light source was connected to a PC. EB1985WU projects a rectangular light at an intensity of 4800 lumens. The color of the PC monitor is set to the brightest green fluorescence (R:148 G:241 B:94) so that the projector produces the brightest green light possible, because human eyes and cameras are most sensitive to green light, according to a professional optics company (Kato Koken, Kanagawa, Japan) (Supplemental Video 1).

The projector is set $200-250 \mathrm{~cm}$ caudal to the mannequin (Figure 1A). The green light is directed to a location for the aerosol spread to be determined (Figure 1B). The scattering of the expelled cake flour is seen as an aerosol in the bright green light, which is caused by the Tyndall effect.

Four 4K resolution video cameras (SONY PXW-Z90, SONY, Tokyo, Japan) were used simultaneously to assess the three-dimensional aerosol dispersal. The video recording settings were gain $18 \mathrm{~dB}$, color temperature $3800 \mathrm{~K}$, and f-stop 4.4. All videos were taken by professional photographers (Support Services Bureau of Photography, Sapporo Medical University of Medicine, Sapporo, Japan). Each video camera was placed at a different location (side and front of the mannequin, side of the physician's face, and at a 45-degree angle from the upper caudal side of the medical staff's face).

\section{Experimental Settings}


The experiments were conducted in a standard clinical operating room (Table 1). The medical staff stood on the patient's right side.

Intubation and extubation were performed with an 8.0-mm Hi-Contour Oral/Nasal Tracheal Tube Cuffed (Covidien, Dublin, $\mathrm{OH}, \mathrm{USA}$ ). The neck of the mannequin during intubation and extubation was retroflexed and in the neutral position, respectively. The artificial cough was expelled when the endotracheal tube was fixed at $16 \mathrm{~cm}$ (the tip of the endotracheal tube reached just the glottis of the mannequin) at the corner of the mouth during intubation and extubation.

\section{Experimental settings}

The experiments were conducted in our standard operating room (Figure 1a and Table 1).

\section{Primary outcomes}

The primary outcomes were the aerosolized particle counts at the three points for one minute just after a simulated cough in four settings during intubation or extubation (with an aerosol box during intubation in a depressurized room (DB) or a pressurized room (PB) and without an aerosol box during intubation in a depressurized room (DN) or a pressurized room (PN).

\section{Secondary outcomes}

The secondary outcomes were particle visualizing images (Figure 1b) and videos during intubation without paralysis and extubation in depressurized and pressurized rooms with an aerosol box.

The entire simulation, including the removal of an aerosol box, was recorded.

\section{Statistical assessments}

Data are expressed as means (standard deviation [SD]) and medians (interquartile range [IQR]) for continuous variables and non-continuous variables, respectively, and analysed with the Shapiro-Wilk test. The data were analysed by analysis of variance or the Kruskal-Wallis test among four groups. Post hoc analysis was done with Tukey-Kramer's test or the Steel-D wass test. $\mathrm{P}<0.05$ was considered significant. The statistical analysis was performed with GraphPad Prism 8.0 (GraphPad Software, La Jolla, CA).

\section{Sample size analysis}

According to our preliminary study, delta $=817$ and $S D=425$ were set as representing a significant difference. Power analysis, conducted using R. version 3.5.3, with $\alpha=0.05$, and $1-\beta=0.9$, showed that 6 
samples were required in each group. Considering clinical variations, $20 \%$ of the estimated sample size was added, and it was decided to take 7 samples in each setting.

\section{Results}

\section{Aerosol exposure for the physician, medical staff, and environment}

The summary of the primary outcomes during intubation and extubation is shown in Figures 2 and 3 , and Supplemental Tables E1 and E2 in the online data supplement, respectively. On post hoc analysis, particle counts of all particle sizes at the three places were significantly lower in DB than in PN during intubation. Particle counts of all particle sizes at the physician and medical staff positions were significantly lower in DB than in PN during extubation. Particle counts of all particle sizes at the medical staff position were lower in DB than in PB during intubation.

\section{Visualization of aerosol dispersal}

The simulations during intubation and extubation in a depressurized or pressurized room are shown in Figure 1, Supplemental Table E3, and Supplemental Video 1 in online data supplement.

Two videos of whole views showed that the cough vector was right upward, and the cough hit the ceiling of the aerosol box diagonally in all settings. Four videos during intubation and extubation in a depressurized room showed that aerosolized particles were dissipated immediately after exiting the aerosol box, whereas in a pressurized room the aerosolized particles remained suspended in the air for 15 $s$ and spread widely around the bed.

\section{i) Aerosol box in a depressurized room}

During intubation, the side whole view showed aerosolized particles spread maximally to $20 \mathrm{~cm}$ caudally and $5 \mathrm{~cm}$ upward out of the aerosol box. The vertical whole view showed that aerosol particles spread maximally to $25 \mathrm{~cm}$ sideways and in the direction of the medical staff out of the aerosol box. The nurse's view showed that the aerosolized particles reached the medical staff's forearm, breast, and face shield. The doctor's view showed that aerosolized particles moved slowly across the external roof of the aerosol box and reached the physician's face.

During extubation, the side whole view showed that aerosolized particles spread maximally to $15 \mathrm{~cm}$ caudally and $5 \mathrm{~cm}$ upward out of the aerosol box. The vertical whole view showed that aerosol particles spread maximally to $15 \mathrm{~cm}$ sideways and in the direction of the medical staff out of the aerosol box. The nurse's view showed that the aerosolized particles reached the medical staff's forearm, breast, and face 
shield. The doctor's view showed that aerosolized particles moved slowly across the external roof of the aerosol box and reached the physician's face.

\section{ii) Aerosol box in a pressurized room}

During intubation, both the physician and the medical staff were exposed to extremely high aerosol levels.

During extubation, the medical staff was exposed to extremely high aerosol levels, whereas the physician's exposure could not be detected. The aerosolized particles spread widely in the whole room during the extubation phase.

In all conditions, aerosol particles that remained in the aerosol box spread with removal of the box. All of the doctor's views showed that the physician got aerosol exposure during removal of the aerosol box. The end of Supplemental Video 1 focused on aerosol dispersal during box removal.

\section{Discussion}

The present results, based on quantitative, temporal, and visual assessments, showed that an aerosol box in a depressurized room significantly decreased physician, medical staff, and environmental aerosol exposure compared with and without an aerosol box in a pressurized room during intubation and extubation. However, an aerosol box in a depressurized room did not completely prevent aerosol dispersal during intubation and extubation.

Of particular importance, aerosol exposure of the physician was not prevented during aerosol box removal in a depressurized room and a pressurized room with all settings.

\section{Why an aerosol box did not completely prevent physician aerosol exposure?}

Particle counting showed that an aerosol box did not completely prevent physician aerosol exposure. The videography showed that the aerosolized particles spread and moved on the external roof of the aerosol box to the physician's face. An aerosol box could change air flow direction and speed and the cough vector and re-spread the aerosol, which is why an aerosol box did not completely prevent physician aerosol exposure. The combined particle counting and three-dimensional visual assessment of the present study could explain this.

\section{Difference in aerosol dispersal between pressurized and depressurized rooms}


The differences in aerosol exposure without an aerosol box at three locations between a pressurized room and a depressurized room during intubation and extubation were not significant. On the other hand, the aerosol exposures of the physician and medical staff with an aerosol box were significantly lower in a depressurized room than in a pressurized room. The particle counts (especially for particle diameters $>1.0$ $\mu \mathrm{m}$ ) were decreased at environmental locations $2 \mathrm{~m}$ from the mouth of the mannequin with or without an aerosol box in a depressurized room during intubation and extubation. The results showed that the depressurized room enhanced the efficacy of the aerosol box, and the medical staff and instruments must be more than $2 \mathrm{~m}$ from the patient's mouth during intubation and extubation in a depressurized room. In a pressurized room, a distance of $2 \mathrm{~m}$ was insufficient to decrease aerosol exposure even with an aerosol box.

In our hospital, the most important difference between a depressurized room and a pressurized room is whether there is an airflow pathway. The results suggest that the airflow pathway is an important factor to spread aerosolized particles. The airflow in a depressurized room can pass into the duct immediately so that the airflow speed can be kept high enough for aerosolized particles (especially $>1.0 \mu \mathrm{m}$ ) to drop. However, the airflow in a pressurized room (in our operating room, the initial airflow speeds in both rooms are the same) could not maintain enough speed for aerosolized particles to drop, because there is no airflow exit in a pressurized room. The association between aerosol dispersal and the airflow pathway is considered important not only in the operating room, but also on patient wards, in emergency rooms, and in intensive care units.

\section{Difference between the present study and other studies}

Although one study has provided visual evidence of two-dimensional aerosol dispersal of a human cough with a face mask,[6] the present study is the first to demonstrate visual aerosol dispersal during intubation and extubation in an operating room under clinical settings. Moreover, the present study provides the first evidence of three-dimensional visual aerosol dispersal during intubation and extubation. Some studies used a strong laser sheet to assess aerosol dispersal and droplets, because a strong laser sheet is useful to dramatically improve the visualization of aerosols compared with other light sources. $[6,7]$ However, visual assessment of aerosol dispersal with a strong laser sheet is not enough to assess spatial aerosol dispersal, because a laser sheet can detect particles only along the laser pathway, providing a two-dimensional assessment of aerosol dispersal. In addition, RIKEN (Kobe, Japan) showed that a supercomputer (Fugaku, RIKEN, Kobe, Japan) can predict spatial aerosol dispersal after cough.[8] However, even supercomputers, which are only available in a few countries, cannot include all clinical and environmental settings and the movements of physicians and medical staff in the prediction. The present experiment required only an ACLS mannequin, cake flour, a projector, and a PC, which are common items. The present study showed a method of assessing aerosol dispersal independently with common items, which is useful. 
Particle counting during intubation and coughing is useful for objective, numerical assessment of aerosol dispersal.[4,9] However, particle counters can count only particles that enter the device inlet. Furthermore, most particle counters cannot measure the full range of aerosol particle sizes simultaneously.[4,9] Although Simpson et al.[4] showed particle numbers with small variation during experimental intubation models, their results were measured in a powerfully depressurized room (pressure -10 Pa, 18 room air changes per hour). Their results apply only under extremely limited conditions. Most critically ill patients must be intubated not in an operating room or intensive care unit, but in an emergency room or general ward during a pandemic.

The present complex assessment that consisted of three-dimensional assessment and particle counting appears necessary and useful to analyse aerosol dispersal.

\section{Experimental settings/tracers}

The range of aerosol diameters was defined as $0.01-100 \mu \mathrm{m}$ by the Centers for Disease Control and Prevention. Cake flour, submicron oil particles, and titanium dioxide have been used as aerosol models in some studies.[10-12] The size of infective aerosol particles was reported to be $>5-10 \mu \mathrm{m}$.[13] Airborne transmission could be attributed to some pathogens, including SARS-CoV-2, with sizes $\leq 5 \mu \mathrm{m}$.[13] According to the present experiments, cake flour $\left(2.5-280 \mathrm{~mm}\left(\mathrm{D}_{50}=25.56 \mu \mathrm{m}\right)\right)$ was suitable as a tracer of aerosolized particles with diameters $>2.5 \mathrm{~mm}$. The present results for particles $<2.5 \mu \mathrm{m}$ in size could be unstable and reference values, because an aerosolized particle is not a perfect sphere.

\section{Pattern of aerosol dispersal during intubation and extubation}

During both intubation and extubation, the aerosol tended to spread to the right side. This can be explained by the laryngoscope having been inserted from the right side during intubation, and the endotracheal tube, which was fixed at the right corner of the mouth, was removed from the right side. Medical staff should not stand on the patient's right side during intubation and extubation.

In the present settings, an aerosol box did not protect physicians and medical staff from aerosol exposure during intubation and removal of the aerosol box even in a depressurized room. This is important, because we must intubate infected patients with paralysis during this pandemic. Because full personal protective equipment (PPE) cannot prevent aerosol transmission completely,[14] neuromuscular blockade is essential for intubation. Moreover, medical personnel should pay attention to aerosol dispersal again with the removal of the aerosol box after the procedures.

\section{Efficacy of an aerosol box}


Unfortunately, the present results did not show the efficacy of an aerosol box at three locations in a pressurized room. An aerosol box in a depressurized room could decrease most particle counts at three locations, but not significantly. Combined use of an aerosol box and a depressurized room significantly decreased particle counts at three locations during intubation and extubation. However, the quantitative and visual results showed that an aerosol box did not completely prevent aerosol exposure of the physician and medical staff.

A recent study showed that an aerosol box effectively prevented aerosol dispersal.[4] However, this study was conducted in a powerfully depressurized room, but such a powerfully depressurized room is not usually available for physicians to use. In most clinical settings (pressurized rooms), an aerosol box cannot be used to prevent aerosol transmission during intubation and extubation.

\section{Environmental settings}

Environmental factors including air change rate,[15] fresh air change rate,[15] room size,[16] temperature, [2] humidity,[2] airflow and turbulence,[15] atmospheric pressure in a depressurized room or pressurized pressure room,[15] bed position, patient's head position during intubation and extubation, and the position and posture of the physician and medical staff could affect aerosol dispersal. All factors could differ among countries, hospitals, and rooms. The present particle visualizing method could allow clinicians to assess aerosol dispersal independently with common items.

\section{Recommendations from the present study to protect from aerosol exposure during intubation and extubation}

i) All aerosol-generating procedures must be done leeward. If necessary, the bed position should be moved considering the direction of airflow. The physicians and medical staff must stand windward. It is better to place any instruments windward. Even if airflow speed is high enough to eliminate aerosolized particles, a duct is necessary and can create an airflow pathway, which is important to eliminate aerosolized particles.

ii) During intubation, medical staff should stand on the patient's left side. During extubation, the medical staff should stand on the patient's left side if the endotracheal tube was fixed at the right corner of the mouth.

iii) Intubation must be done with neuromuscular blockade, even in a depressurized room and with full PPE and an aerosol box.

iv) Extubation could be done alone as well as possible even in a depressurized room. An aerosol box cannot completely prevent aerosol dispersal during extubation. 
v) Independent assessment of aerosol dispersal is essential to protect patients, medical staff, and physicians from aerosol exposure. The present particle visualizing method can be used to assess threedimensional aerosol dispersal with common items (if video cameras are unavailable, naked eye observation may be sufficient).

vi) If an aerosol box is used during intubation and extubation, attention to aerosol dispersal is again needed when removing it.

\section{Limitations}

As a limitation, the diameter of the cake flour was 5-100 mm, which results in a somewhat artificial human coughing pattern because of the aerodynamics (Supplemental Video 1),[17] since differences in particle diameters and coughing patterns affect aerosol spread.[17] Because cake flour does not include small aerosolized particles $(0.01-1 \mu \mathrm{m})$, and even high-resolution cameras cannot detect all sizes of aerosolized particles, clinical aerosol exposure could be greater than in the current experiment.

\section{Conclusions}

The present findings showed that an aerosol box decreased aerosol exposure of physicians, medical staff, and the environment only in a depressurized room. The three-dimensional particle visual assessment with common items provides an independent aerosol dispersal assessment that can be used worldwide and is useful to assess aerosol dispersal under usual clinical conditions.

\section{Declarations}

Funding:

No

\section{Conflicts of interest/Competing interests:}

No

\section{Ethics approval:}

Not applicable

\section{Consent to participate:}

Not applicable 


\section{Consent for publication:}

Not applicable

\section{Availability of data and material:}

All data generated or analysed during this study are included in this published article and its supplementary information files.

\section{Authors' contributions:}

Wataru Sakai: This author planned the study and wrote the manuscript.

Gen Hasegawa: This author helped our experiments.

Tomohiro Chaki, Shunsuke Tachibana and Michiaki Yamakage: These authors helped write and review the manuscript.

\section{Acknowledgements:}

The authors are grateful to Kato Koken (Kanagawa, Japan) and HORIBA, Ltd. (Kyoto, Japan) for collaborating with this work at no cost.

\section{Take Home Message:}

An aerosol box decreased aerosol exposure of the physician, medical staff, and environment only in a depressurized room. Our three-dimensional particle visual assessment with common items provides an independent, clinical aerosol dispersal assessment.

\section{References}

1. Tang YW, Schmitz JE, Persing DH et al (2020) Laboratory diagnosis of COVID-19: Current issues and challenges. J Clin Microbiol 58:e00512-20.

2. Elazhary MA, Derbyshire JB (1979) Effect of temperature, relative humidity and medium on the aerosol stability of infectious bovine rhinotracheitis virus. Can J Comp Med 43:158-167.

3. Canelli R, Connor CW, Gonzalez M et al (2020) Barrier enclosure during endotracheal intubation. N Engl J Med 382:1957-1958.

4. Simpson JP, Wong DN, Verco L et al (2020) Measurement of airborne particle exposure during simulated tracheal intubation using various proposed aerosol containment devices during the 
COVID-19 pandemic. Anaesthesia 75:1587-1595.

5. Begley JL, Lavery KE, Nickson CP et al (2020) The aerosol box for intubation in coronavirus disease 2019 patients: an in-situ simulation crossover study. Anaesthesia 75:1014-1021.

6. Verma S, Dhanak M, Frankenfield J (2020) Visualizing the effectiveness of face masks in obstructing respiratory jets. Phys Fluids (1994) 32:061708.

7. Anfinrud P, Stadnytskyi V, Bax CE et al (2020) Visualizing speech-generated oral fluid droplets with laser light scattering. N Engl J Med 382:2061-2063.

8. https://www.r-ccs.riken.jp/outreach/formedia/200603Tsubokura. Accessed 1 Nov 2020

9. Burkart J, Steiner G, Reischl G et al (2010) Characterizing the performance of two optical particle counters (Grimm OPC1.108 and OPC1.109) under urban aerosol conditions. J Aerosol Sci 41:953962.

10. Stobnicka A, Górny RL (2015) Exposure to flour dust in the occupational environment. Int J Occup Saf Ergon 21:241-249.

11. Fu H, Patel AC, Holtzman MJ et al (2011) A new electrospray aerosol generator with high particle transmission efficiency. Aerosol Sci Technol 45:1176-1183.

12. Pujalté I, Serventi A, Noël A et al (2017) Characterization of aerosols of titanium dioxide nanoparticles following three generation methods using an optimized aerosolization system designed for experimental inhalation studies. Toxics 5:14.

13. Fennelly KP (2020) Particle sizes of infectious aerosols: implications for infection control. Lancet Respir Med 8:914-924.

14. Perencevich EN, Diekema DJ, Edmond MB (2020) Moving personal protective equipment into the community: face shields and containment of COVID-19. JAMA 323:2252-2253.

15. Vuorinen V, Aarnio M, Alava M et al (2020) Modelling aerosol transport and virus exposure with numerical simulations in relation to SARS-CoV-2 transmission by inhalation indoors. Safety science. https://doi.org/10.1016/j.ssci.2020.104866

16. Bello A, Quinn MM, Perry MJ et al (2010) Quantitative assessment of airborne exposures generated during common cleaning tasks: a pilot study. Environ Health 9: 76.

17. Ciofi-Silva CL, Hansen LL, Almeida AG et al (2016) Negative pressure of the environmental air in the cleaning area of the materials and sterilization center: a systematic review. Rev Lat Am Enfermagem 24: e2781.

18. Fabriès JF, Choudat D, Wrobel R et al (2000) Computerized equipment for the delivery of inhaled doses of solid particles in specific bronchial challenge. J Aerosol Med 13: 1-10.

\section{Figures}


A
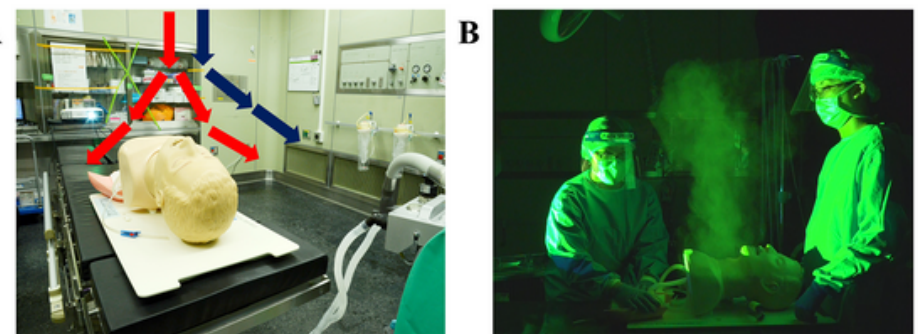

C

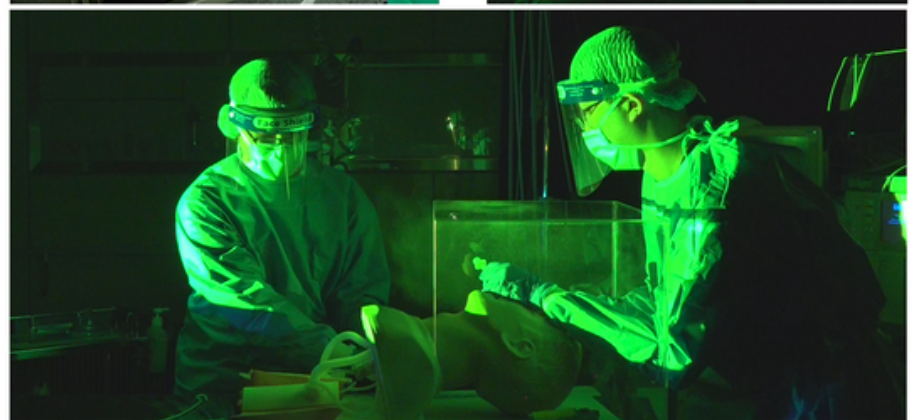

D

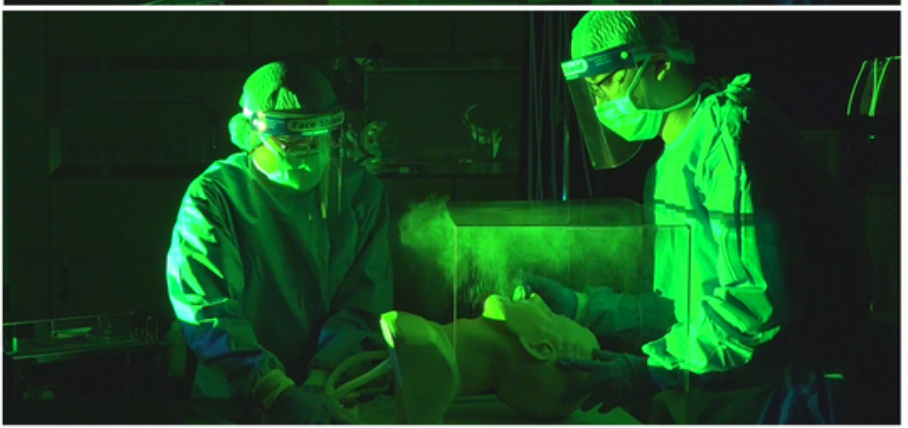

E

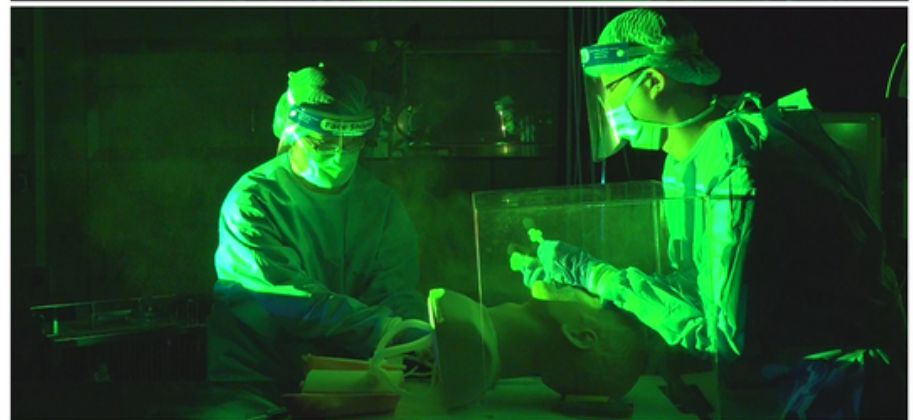

F

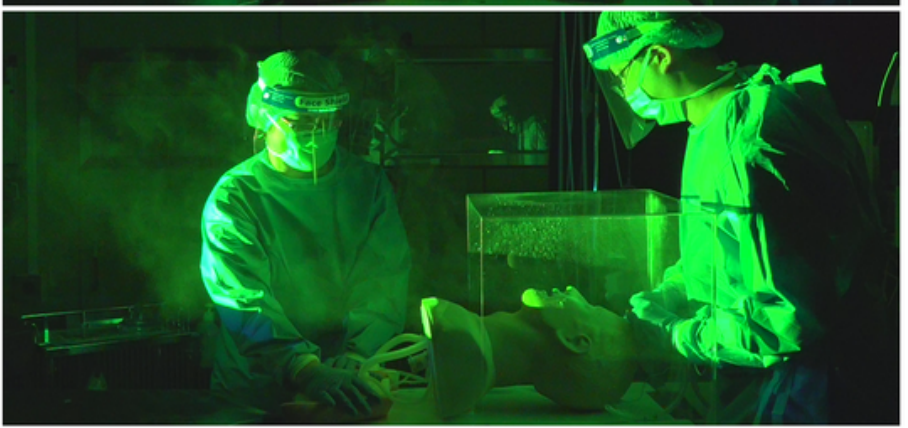

Figure 1

Preparation and set up of the experiment to simulate aerosol spread during intubation and extubation with an aerosol box in depressurized and pressurized rooms Neck position for intubation or extubation in a depressurized room or a pressurized room can affect the pattern of aerosol spread. (a) The preparation and set up of the simulation. The red and blue arrows show the wind directions in the depressurized and pressurized rooms, respectively. A vent can only be used in a depressurized room. (b) The brightest green 
light is directed to a location for the aerosol spread to be determined. (c) Aerosol spread during intubation without paralysis in a depressurized room. The physician (right) is protected from the expelled aerosolized particles, which dissipate after exiting the aerosol box, but not the medical staff (left). (d) Aerosol spread during extubation in a depressurized room. The physician (right) is protected from the expelled aerosolized particles, which dissipate after exiting the aerosol box, but not the medical staff (left). (e) Aerosol spread during intubation without paralysis in a pressurized room. The physician (right) is protected from the expelled aerosolized particles, but the medical staff (left) is highly exposed, as the expelled aerosolized particles spread widely. (f) Aerosol spread during extubation in a pressurized room. The physician (right) is protected from the expelled aerosolized particles, but the medical staff (left) is highly exposed. 
I

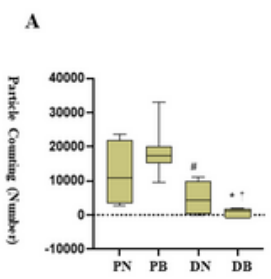

D

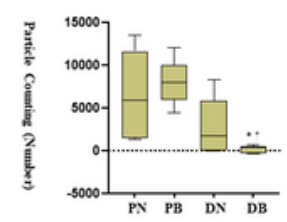

II
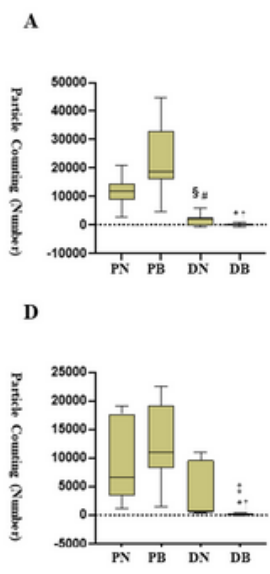

III

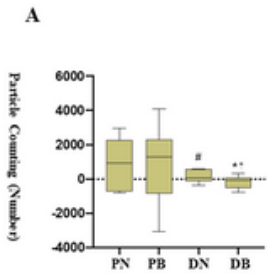

D

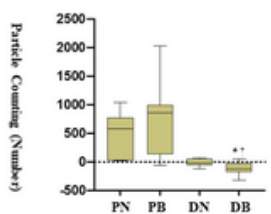

B
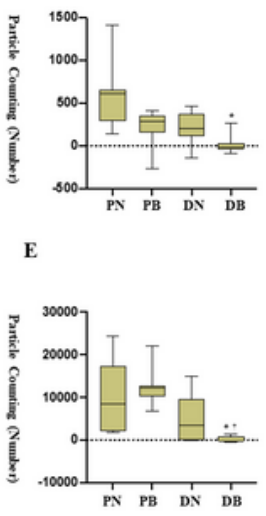

B

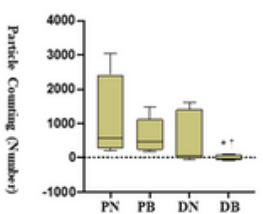

E

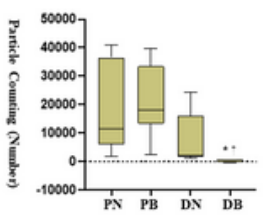

B

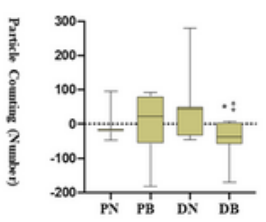

E

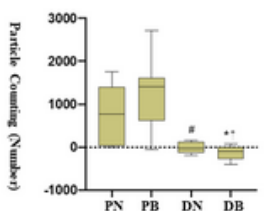

C

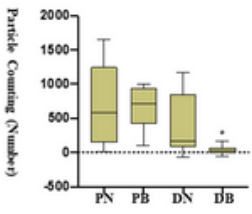

F

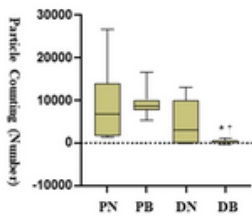

C
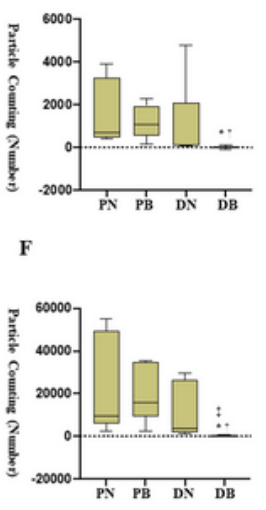

C
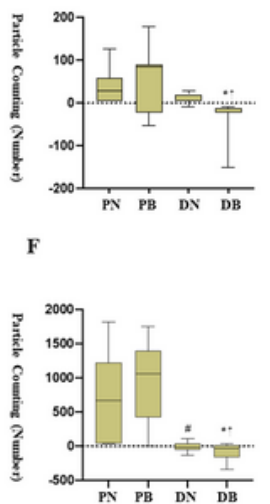

\section{Figure 2}

Summary of particle counts during intubation. (I) Physician's aerosol exposure (II) Medical staff's aerosol exposure (III) Environmental aerosol exposure, A $0.3 \mu \mathrm{m}, \mathrm{B} 0.5 \mu \mathrm{m}, \mathrm{C} 1.0 \mu \mathrm{m}, \mathrm{D} 2.5 \mu \mathrm{m}, \mathrm{E} 5.0 \mu \mathrm{m}$, *significant difference between $\mathrm{DB}$ and $\mathrm{PN}, \mathrm{t}$-significant difference between $\mathrm{DB}$ and $\mathrm{PB}$, $\ddagger$-significant difference between DB and DN, \#-significant difference between PB and DN, §-significant difference between PN and DN, PN-in a pressurized room without an aerosol box, PB-in a pressurized room with an 
aerosol box, DN-in a depressurized room without an aerosol box, DB-in a depressurized room with an aerosol box

I
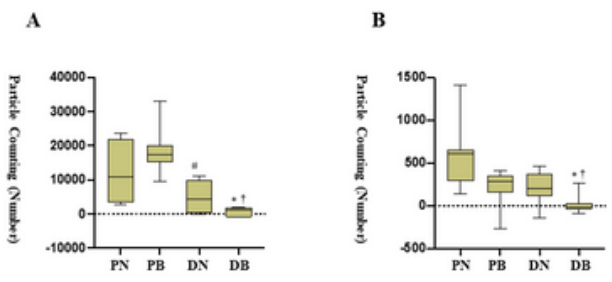

D

E
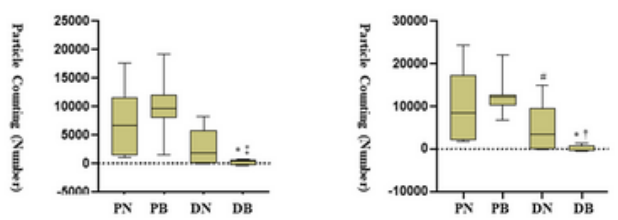

II

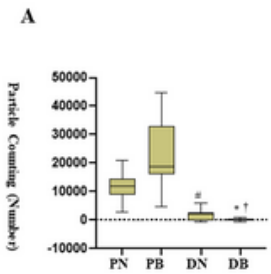

B

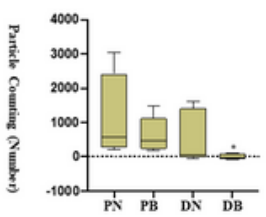

$\mathbf{D}$

$\mathbf{E}$

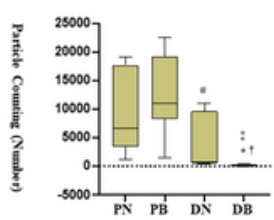

III

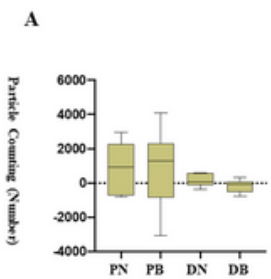

D
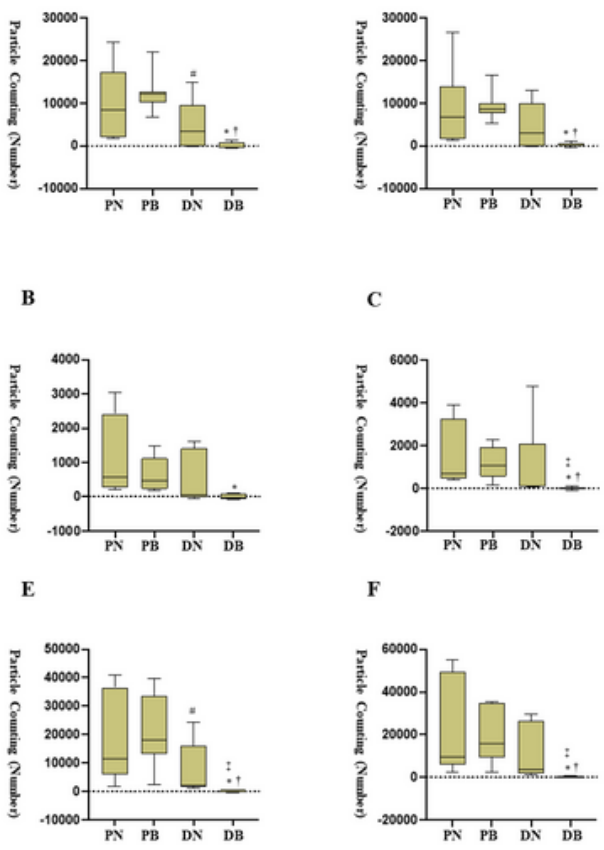

$\mathbf{F}$
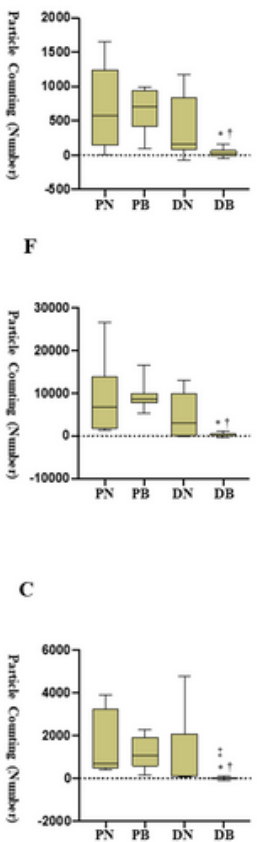

$\mathbf{F}$
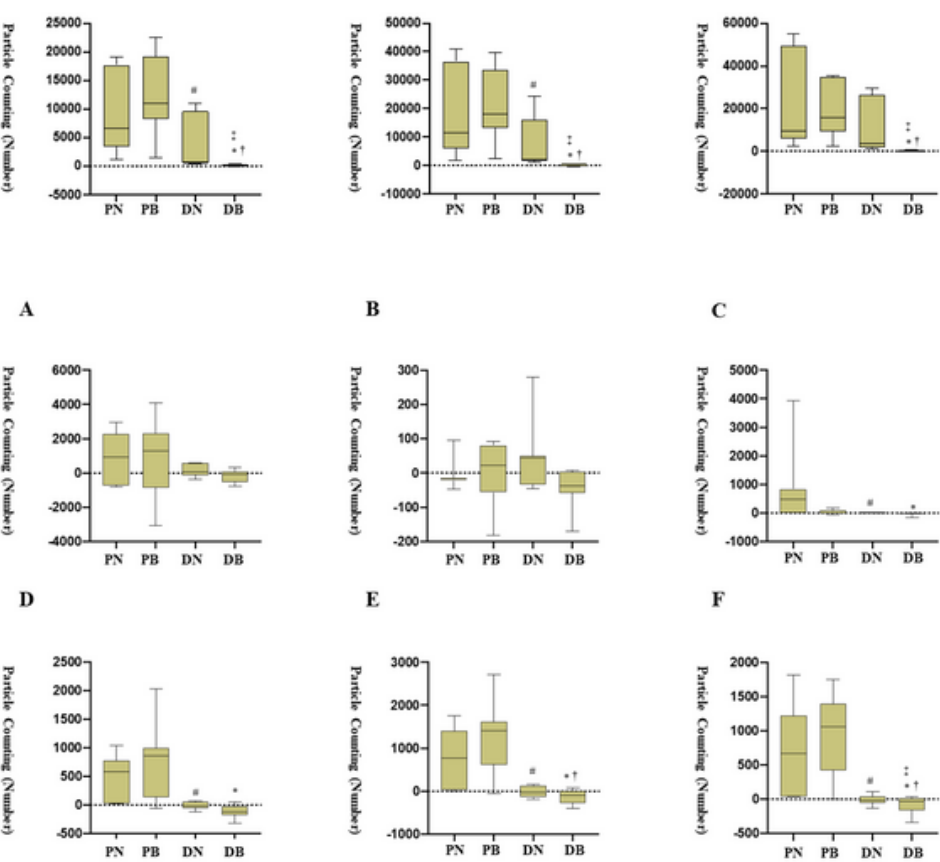

C

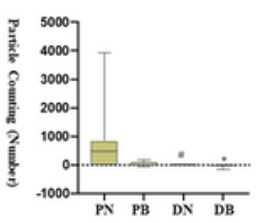

E

F
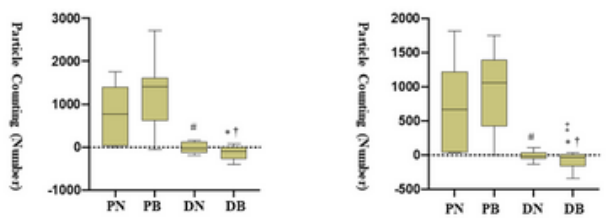

Figure 3

Summary of particle counts during extubation. (I) Physician's aerosol exposure (II) Medical staff's aerosol exposure (III) Environmental aerosol exposure, A $0.3 \mu \mathrm{m}, \mathrm{B} 0.5 \mu \mathrm{m}, \mathrm{C} 1.0 \mu \mathrm{m}, \mathrm{D} 2.5 \mu \mathrm{m}, \mathrm{E} 5.0 \mu \mathrm{m}$, *significant difference between DB and PN, †-significant difference between DB and PB, $\neq$-significant 
difference between DB and DN, \#-significant difference between PB and DN, §-significant difference between $\mathrm{PN}$ and $\mathrm{DN}, \mathrm{PN}$-in a pressurized room without an aerosol box, $\mathrm{PB}$-in a pressurized room with an aerosol box, DN-in a depressurized room without an aerosol box, DB-in a depressurized room with an aerosol box

\section{Supplementary Files}

This is a list of supplementary files associated with this preprint. Click to download.

- SupplementalTableE123andSupplementalVideo1.docx

- SupplementalVideo15min40sec1.mp4 